\title{
In vivo Human 3D Cardiac Fibre Architecture: Reconstruction Using Curvilinear Interpolation of Diffusion Tensor Images
}

\author{
Nicolas Toussaint ${ }^{1,2}$, Maxime Sermesant ${ }^{1,2}$, Christian T. Stoeck ${ }^{3}$, \\ Sebastian Kozerke ${ }^{1,3}$, and Philip G. Batchelor ${ }^{1}$ \\ 1 King's College London, Imaging Sciences, London, UK \\ nicolas.toussaint@kcl.ac.uk \\ ${ }^{2}$ INRIA, Asclepios Research Group, Sophia Antipolis, France \\ 3 ETH Zürich, Institute for Biomedical Engineering, Switzerland
}

\begin{abstract}
In vivo imaging of the cardiac 3D fibre architecture is still a challenge, but it would have many clinical applications, for instance to better understand pathologies and to follow up remodelling after therapy. Recently, cardiac MRI enabled the acquisition of Diffusion Tensor images (DTI) of 2D slices. We propose a method for the complete 3D reconstruction of cardiac fibre architecture in the left ventricular myocardium from sparse in vivo DTI slices. This is achieved in two steps. First we map non-linearly the left ventricular geometry to a truncated ellipsoid. Second, we express coordinates and tensor components in Prolate Spheroidal System, where an anisotropic Gaussian kernel regression interpolation is performed. The framework is initially applied to a statistical cardiac DTI atlas in order to estimate the optimal anisotropic bandwidths. Then, it is applied to in vivo beating heart DTI data sparsely acquired on a healthy subject. Resulting in vivo tensor field shows good correlation with literature, especially the elevation (helix) angle transmural variation. To our knowledge, this is the first reconstruction of in vivo human $3 \mathrm{D}$ cardiac fibre structure. Such approach opens up possibilities in terms of analysis of the fibre architecture in patients.
\end{abstract}

\section{Introduction}

Cardiac fibre architecture has a crucial role in the cardiac function, as it influences heavily the muscle electrophysiological and mechanical behaviours. For instance electrical propagation is three times faster in the fibre direction than in the orthogonal plan [8]. For such reasons its study can have an important impact on clinical decisions, as several cardiac pathologies - such as myocardial infarction, cardiomyopathy, hypertension, or valvular heart diseases - involve a rearrangement of myocardial fibres [16].

Diffusion Tensor MRI (DTI) can depict non-invasively the fibre orientation distribution of the myocardium 61417]. Moreover, taking into account the full information given by the tensors gives an insight on the intrinsic laminar sheet 
structure [12]. However, using this technique in-vivo is challenging: complex motion patterns due to heartbeat creates important distortions of the signal. Furthermore, the muscle tissue has relatively low anisotropy. Recent studies however raised a new hope [2 5], but the difficulty and sensitivity of these techniques limit the data that could be acquired in clinical time, resulting in sparsely distributed acquisitions.

We set up a method to interpolate the in vivo Left Ventricule (LV) sparse tensor data in a shape-adapted curvilinear coordinate system (i.e. Prolate Spheroidal System, PSS). We explain how we non-linearly register the LV to an ellipsoid to express a Kernel regression scheme in PSS. Spatial coherence of a statistical cardiac DTI atlas is used as a prior for our curved interpolation. By mapping back the resulting tensor information to the $\mathrm{LV}$, we are able to reconstruct the complete tensor structure of the LV, and compare it with those obtained with Cartesian interpolation.

\section{Curvilinear Interpolation of Tensors}

\subsection{Truncated Prolate Spheroid 3D Diffeomorphic Mapping}

The shape of the LV has been assimilated to a truncated ellipsoid [19], on which we can use the shape adapted curvilinear coordinate system called Prolate Spheroidal System [15] (PSS). Note that this coordinate system is already used to describe mechanical behaviour of the heart 119.

A 3D binary mask of the left ventricle is manually delineated on a 3D MRI of the heart, and registered to a 3D synthetic binary mask of a perfect truncated prolate spheroid (see Fig. 1, c). In order to have an invertible and symmetric mapping of positions, we chose a symmetric version of the Diffeomorphic Demons registration algorithm [3]. In addition, an elasticity contraint, as introduced in 10. has been incorporated to ensure a smooth displacement of the middle of the ventricle wall. The advantage of this method is that it provides us with smooth and elastic displacement fields, from the LV to the spheroid and vice versa.

In this setting, any acquired tensor $D_{x}$ lying within the LV wall is warped using the forward transformation $\Phi$. We reorient it using the Finite Strain strategy as it seems well suited to preserve geometric features of cardiac diffusion tensor fields [12], with the Jacobian of the backward transformation $\Phi^{-1}$.

PSS is well adapted to the LV shape 9] as local coordinates have physiological meaning (see Fig. 1 (b)): $\xi_{1}$ is the positive transmural abcissa, $\xi_{2}$ is the apex-base abcissa from 0 to $\pi / 2$, and $\xi_{3}$ is the circumferential abcissa from 0 to $2 \pi$. Thus, once the tensor data is mapped on the prolate spheroid, we can compute PSS coordinate position vectors $\left(\xi_{1}, \xi_{2}, \xi_{3}\right)$ of each measure point, and describe tensors components in local contravariant basis $\mathcal{B}_{\xi}=\left(g_{1}, g_{2}, g_{3}\right)$. The transformation between basis are then written: $D_{\xi}=\mathcal{B}_{\xi}^{T} . \Phi\left(D_{x}\right) \cdot \mathcal{B}_{\xi}$ and $D_{x}=\Phi^{-1}\left(\mathcal{B}_{\xi} \cdot D_{\xi} \cdot \mathcal{B}_{\xi}^{T}\right)$.

\subsection{Anisotropic Kernel Estimator in Curvilinear Coordinates}

Let us consider the situation where we have a set of $N$ known tensors $\left(D_{\xi_{i}}\right)_{1}^{N}$ over the domain of interest $\Omega^{\prime}$, which is the image of the LV: $\Omega^{\prime}=\Phi(\Omega)$. 


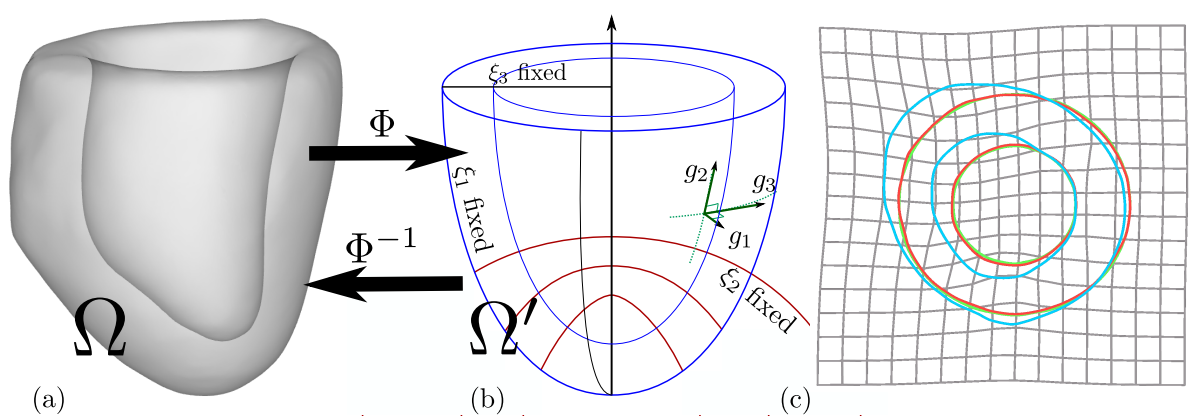

Fig. 1. 3D Mapping of a binary mask of the LV (a) to a synthetic prolate spheroid (b) using adapted symmetric diffeomorphic demons. Transformations $\Phi$ and $\Phi^{-1}$ are smooth and inverse of each other. (b) shows level-sets of PSS coordinates, as well as the local contravariant basis vectors $\left(g_{1}, g_{2}, g_{3}\right)$. (c) demonstrates the quality of the 3D registration. The $\mathrm{LV}$ (blue) is registered towards the synthetic spheroid (green). The registered $\mathrm{LV}$ outline (red) fits with its target with a smooth displacement (grey).

We want to estimate the missing tensor $D_{\xi}$ at position $\xi$. Assuming that the tensor field is continuous and relatively homogeneous in the myocardium, we can claim that $D_{\xi}$ is directly influenced by its neighbours. In this context we use a Gaussian kernel estimator $K_{\sigma}$ of bandwidth $\sigma$. The kernel estimate $\hat{m}_{\sigma}\left(D_{\xi}\right)$ is taken as the Log-Euclidean [4] weighted mean of surrounding tensors, and thus written:

$$
\hat{m}_{\sigma}\left(D_{\xi}\right)=\exp \left(\frac{\sum_{i=1}^{N} K_{\sigma}\left(\xi-\xi_{i}\right) \log \left(D_{\xi_{i}}\right)}{\sum_{i=1}^{N} K_{\sigma}\left(\xi-\xi_{i}\right)}\right)
$$

PSS coordinates are highly non-homogeneous, therefore an anisotropic interpolation with respect to each of the coordinates is needed. We thus replace the scalar $\sigma$ by a positive definite matrix $H$. Let $X$ be the position in a given coordinate system as a column vector $\left(X_{1}, X_{2}, X_{3}\right)^{T}$ and let $d X=\left(X-X_{i}\right)$. The kernel becomes [7:

$$
K_{H}: d X \rightarrow K_{H}(d X)=\operatorname{det}(H)^{-1} K\left(\sqrt{d X^{T} H^{-2} d X}\right)
$$

where $K$ is the univariate kernel function (we use Normal Gaussian function). $H$ is invertible as positive definite. The difficulty remains in the choice over the matrix $H$. We follow ideas from Härdle in [7] and define a rule to find optimal bandwidth $H_{o p t}$ to be used. It consists in a Leave-One-Out approach where we minimize residual norm (or Least Square LS). In the next section we estimate appropriate bandwidth matrices, in both PSS and Cartesian coordinate systems, using a cardiac DTI atlas.

\section{Parameter Estimation Using a Cardiac DTI Atlas}

In order to estimate the interpolation parameters, we took advantage of a publicly available cardiac DTI atlas of canine hearts [12. Our study is limited to the 
left ventricle in order to match with the in vivo data studied in the next section. If we only know partial data $\left(D_{\xi_{i}}\right)_{1}^{N}$ on the atlas, let $\alpha$ be the data coverage ratio between the known data $N$ and the total number of voxels of the atlas LV $M$ : $\alpha=N / M$. To reproduce in-vivo acquisition environment, The atlas is divided slice by slice in the long axis direction (i.e. imitating sparsely distributed short axis slices). Normal distribution noise (of variance $V=0.01$ ) was added over the tensor's corresponding DWIs. We propose to find the optimal bandwidth $H_{o p t}$ that minimizes the LS criterion over the entire ventricle domain $\Omega$. We are looking for a trade-off between fit quality and smoothness, so we minimize the criterion:

$$
C V(H)=\underbrace{\sum_{x_{j} \in \Omega} \operatorname{dist}\left(D_{x_{j}}, \hat{m}_{H}\left(D_{x_{j}}\right)\right)^{2}}_{\text {data fit } L S(H)}+\lambda \cdot \underbrace{\sum_{x_{j} \in \Omega}\left\|\nabla \hat{m}_{H}\left(D_{x_{j}}\right)\right\|^{2}}_{\text {smoothness } \operatorname{Reg}(H)}
$$

where the distance dist is taken in Log-Euclidean metrics, i.e. the Frobenius norm of the $\log \operatorname{difference} \operatorname{dist}(A, B)=\|\log (A)-\log (B)\|$. The criterion $C V(H)$ to be minimized thus includes a scalar $\lambda$ to control the regularization. We chose to use a gradient-free multivariate optimization scheme derived from Powell \& al. [13] to minimize $C V(H)$. Furthermore, $H$ was constrained to be diagonal. Note that optimal bandwidth matrices are different in PSS interpolation $\left(H_{o p t}^{p}\right)$ and in Cartesian interpolation $\left(H_{o p t}^{c}\right)$.

We ran the minimization process for several values of $\lambda$ to find a suitable regularization ratio. Fig. 2, a shows a log-log scatter graph of the regularization term vs. the data fit term as result of the L-curve in PSS coordinates. We see an inflexion point of maximal curvature at $\lambda_{o p t}=1.5$ for a coverage of $\alpha=60 \%$. We used this value of $\lambda$ for the remaining of this paper.

The long axis data coverage ratio $\alpha$ has a physical meaning: in practice, a full coverage of the ventricle is of great difficulty and in vivo data is often limited to short axis views around the equatorial region. We thus reproduced the optimization experiment varying $\alpha$. The evolution of $C V\left(H_{o p t}\right)$ against $\alpha$ is shown in Fig. 2. b. At low data coverage the quality of data fit using PSS interpolation is more than $100 \%$ better compared to Cartesian interpolation. Moreover we achieve a very good fit after $50 \%$ of coverage, while it is only above $80 \%$ that we have similar fit with both coordinate systems.

The eigenvalues of $H_{o p t}$ (bandwidths in each axis direction) are of great importance as they will be used as spatial coherence prior for the in vivo study. They depend on the noise level as well as the data coverage ratio of the ventricle. Fig. 3] summarizes the variation of these optimal bandwidths against the $\alpha$ ratio, using respectively Cartesian and PSS coordinate systems for interpolation. As the ventricle is aligned to the z-axis, a decrease of its corresponding $H_{\text {opt }}$ eigenvalue in Cartesian coordinates can be seen (Fig. 3. a). Cartesian bandwidths at full coverage converge to a value of $0.9 \mathrm{~mm}$. In the PSS coordinate case (33b), optimal bandwidths in both $g_{2}$ and $g_{3}$ directions are decreasing while the one corresponding to $g_{1}$ is stable. They converge respectively to $4 \%$ of wall thickness, 3.4 deg. in apex-base direction, and $6.3 \mathrm{deg}$. in circumferential direction. Now that we 

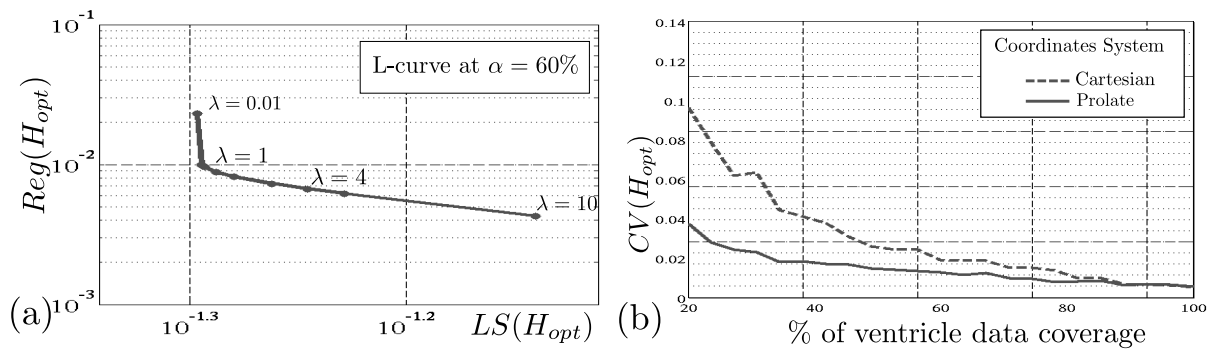

Fig. 2. (a): L-curve of the regularization parameter $\lambda$ in the PSS case for a data coverage ratio $\alpha=60 \%$. The curve shows an inflexion point at $\lambda_{\text {opt }}=1.5$. (b): Evolution of the criterion $C V\left(H_{o p t}\right)$, using $\lambda=\lambda_{o p t}$, against the data coverage ratio $\alpha$. We compare the PSS interpolation performance (solid) versus Cartesian interpolation one (dashed). We see better fit to data in PSS interpolation, especially for low values of $\alpha$.

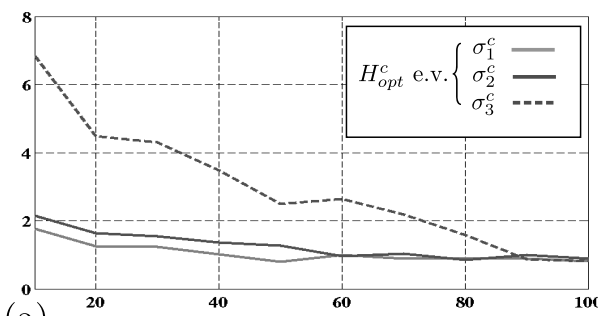

(a)

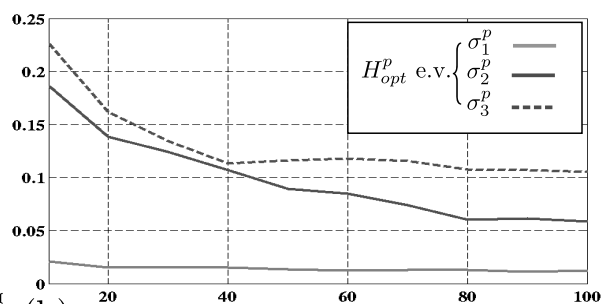

(b)

Fig. 3. $H_{\text {opt }}$ eigenvalues obtained function of the data coverage $\alpha$, using Cartesian interpolation (a), or PSS interpolation (b). While $\sigma_{1}^{c}$ and $\sigma_{2}^{c}$ (corresponding to $\mathrm{x}$ and $\mathrm{y}$ directions) stay stable, $\sigma_{3}^{c}$ (z direction) decreases as the long-axis data resolution raises. Note also the stability of $\sigma_{1}^{p}$ which corresponds to the transmural direction.

estimated optimal bandwidth parameters for our curvilinear interpolation, we can apply this framework to in-vivo data.

\section{Application to in-vivo Human Data}

Multi-slice cardiac DTI data were acquired on a healthy volunteer using a singleshot spin echo sequence on a 3T Philips Achieva System (Philips Healthcare, Best, The Netherlands) with reduced field-of-view excitation and flow compensated diffusion encoding gradients [5]. A total of thirty-one LV short-axis DTI views were obtained around the equatorial level of the left ventricle. The data was acquired in seven separate sessions on the same subject, totalling one hour and thirty minutes of effective scanning. Image acquisition was triggered to peak systole to account for strain effects and utilise maximum myocardial thickness [5]. To account for inconsistencies in volunteer and breath-hold positions among the separate scans, 3D misalignment was corrected using the Insight ToolKit (http://www.itk.org). An additional anatomical $3 \mathrm{D}$ dataset was acquired at the same trigger delay (TD 
$=300 \mathrm{~ms}$ ) as the DTI scans. This anatomical dataset was used to define the left ventricle binary mask to be mapped on the prolate spheroid.

Fig. 4.a shows the input data distribution over the ventricle. Most of the acquisitions have been done in the equatorial part of the LV, because cardiac motion (especially rotation) is of less intensity in this region. We applied the full method detailed above to interpolate tensor data over the entire LV area both using Cartesian coordinates and PSS coordinates. We used the optimal bandwidths obtained at the same coverage level in the atlas study. In that sense we are using the spatial coherence of the atlas as a prior information rather than the atlas tensor data itself. Fig. 4.b shows the evolution of fit to data over the number of slices taken into account. The graph demonstrates better performance of PSS interpolation.
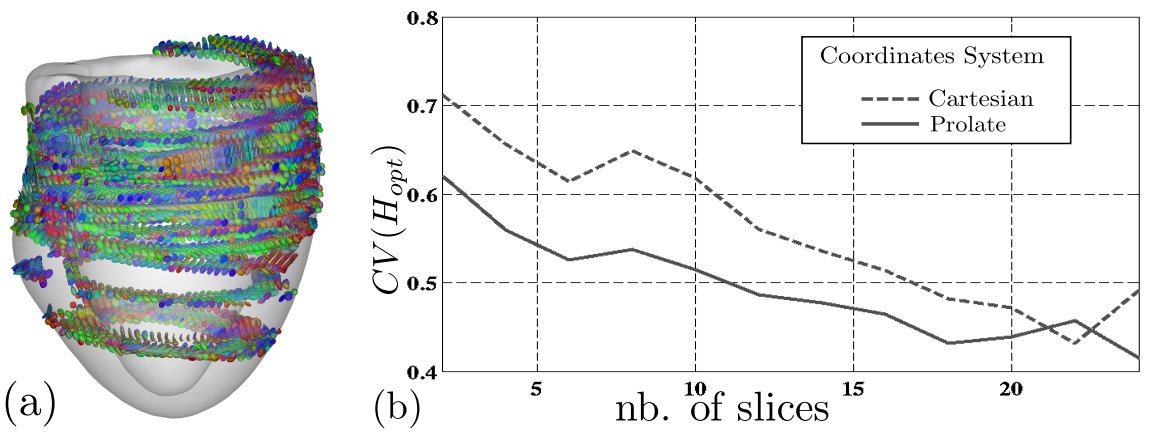

Fig. 4. The in vivo DTI 2D slices were acquired at different occasions (they are not all parrallel to each other), and registered to the anatomical left ventricle. We show tensors color-coded by their first eigenvector direction superimposed with the ventricle outline in (a): The lack of data around the apex is visible. (b) compares performances of interpolation in Cartesian (dashed) and PSS (solid) case against the number of slices.

The helix angle is the angle that the tensor first eigenvector makes with the $\left(g_{1}, g_{3}\right)$ plane (see Fig. 11). The variation of this angle from endocardium to epicardium is an important characteristic of the cardiac function, clearly seen in the DTI atlas and crucial in heart modelling. We show in Fig. 5]joint histograms of the angle against the normalized transmural distance from endocardium to epicardium. The pattern of this variation is reproduced when PSS Coordinates are used, whereas it is less visible in the Cartesian case. Fig. [5] shows a selected slice resulting from in vivo DTI data using Cartesian (Fig. 5,a) and PSS (Fig. 5.b). Tensors are color-coded with the main eigenvector direction. The regularization follows the ventricle shape in the PSS case and thus shows more plausible fibre directions. Finally we were able to track fibres (Fig. 5]c) from these tensors using streamline algorithm. The in vivo fibres have a general circumferential pattern and we can recognize the main helical layers of the endocardium and the epicardium. 

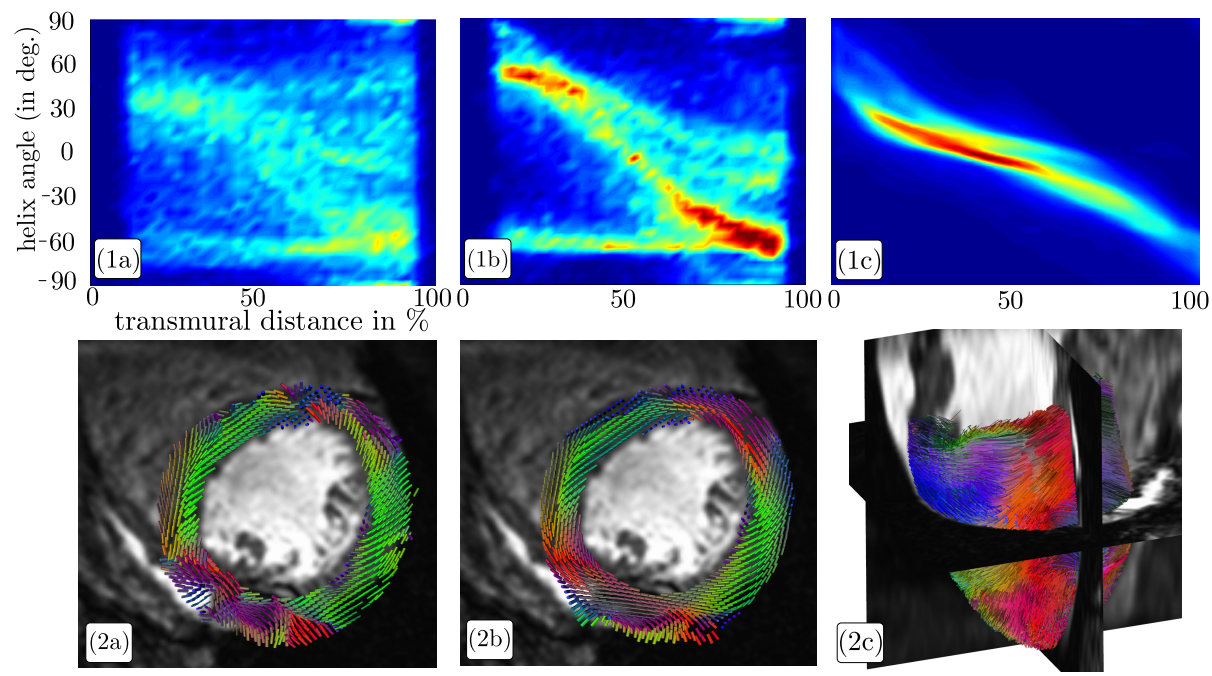

Fig. 5. Top: Joint histograms of the elevation (or helix) angle and the normalized transmural distance from endo to epi. (1a) in-vivo interpolated results using Cartesian coordinates, (1b) in-vivo interpolated results using PSS coordinates, and (1c) as a reference, the fully sampled LV statistical atlas. The correlation is visible using PSS coordinates. Bottom: Interpolated DTI slice color coded by eigenvector direction, using Cartesian Coordinates (2a) and PSS (2b). (2c) is a streamline fibre tractography result from the PSS interpolated tensor field.

\section{Conclusions}

In this paper, we demonstrated that shape adapted curvilinear coordinates - Prolate Spheroidal - are appropriate for the tensor reconstruction over the left ventricle wall volume. We set up a mathematical framework for the kernel regression of tensor data in those coordinates, using anisotropic kernel regression with an optimised bandwidth matrix. We have shown that the resulting interpolated tensors better fit the physionomy of the heart. As the left ventricle has a very characteristic ellipsoidal shape, its fibre architecture (and thus the underlying tensor field) has an important spatial coherence in PSS coordinates, whereas it is less sensible in Cartesian coordinates. We have shown that using the PSS anisotropic spatial coherence of a statistical cardiac DTI atlas as a prior information for in vivo tensor interpolation and regularization helps us to reconstruct full tensor information. We applied our method to reconstruct the fibre architecture of the left ventricle of a healthy volunteer, and, to the best of our knowledge, it is the first time that the in vivo human $3 \mathrm{D}$ structure of the heart has been reconstructed. The in vivo results show a good correlation with literature values of ex vivo human studies. We were able to reproduce the typical pattern of transmural variation of the helix angle. The presented approach opens up possibilities in terms of analysis of the fibre architecture in patients. 


\section{References}

1. Dieudonne, J.M.: The left ventricle as confocal prolate spheroids. Bulletin of Mathematical Biology 31(3), 433-439 (1967)

2. Dou, J., Tseng, W.Y.I., Reese, T.G., Wedeen, V.J.: Combined diffusion and strain MRI reveals structure and function of human myocardial laminar sheets in vivo. Magnetic Resonance in Medicine 50, 107-113 (2003)

3. Dru, F., Vercauteren, T.: An ITK implementation of the symmetric log-domain diffeomorphic demons algorithm. Insight Journal -2009 January - June 2009 (May 2009)

4. Fillard, P., Arsigny, V., Pennec, X., Ayache, N.: Clinical DT-MRI estimation, smoothing and fiber tracking with log-Euclidean metrics. IEEE Transactions on Medical Imaging 26(11), 1472-1482 (2007); pMID: 18041263

5. Gamper, U., Boesiger, P., Kozerke, S.: Diffusion imaging of the in vivo heart using spin echoes - considerations on bulk motion sensitivity. Magnetic Resonance in Medicine 57, 331-337 (2007)

6. Garrido, L., Wedeen, V.J., Kwong, K.K., Spencer, U.M., Kantor, H.L.: Anisotropy of water diffusion in the myocardium of the rat. Circulation Research 74, 789-793 (1994)

7. Härdle, W.K., Marron, J.S.: Optimal bandwidth selection in nonparametric regression function estimation. Annals of Statistics 13(4), 1465-1481 (1985)

8. Kanai, A., Salama, G.: Optical mapping reveals that repolarization spreads anisotropically and is guided by fiber orientation in guinea pig hearts. Circulation Research 77, 784-802 (1995)

9. LeGrice, I.J., Takayama, Y., Covell, J.W.: The architecture of the heart: a databased model. Philosophical Transactions: Mathematical, Physical and Engineering Sciences 359, 1217-1232 (2001)

10. Mansi, T., Peyrat, J.M., Sermesant, M., Delingette, H., Blanc, J., Boudjemline, Y., Ayache, N.: Physically-constrained diffeomorphic demons for the estimation of 3d myocardium strain from cine-MRI. In: Ayache, N., Delingette, H., Sermesant, M. (eds.) FIMH 2009. LNCS, vol. 5528, pp. 201-210. Springer, Heidelberg (2009)

11. Nielsen, P.M., Grice, I.J.L., Smaill, B.H., Hunter, P.J.: Mathematical model of geometry and fibrous structure of the heart. Am. J. Physiol. Heart Circ. Physiol. 260, 1365-1378 (1995)

12. Peyrat, J.M., Sermesant, M., Pennec, X., Hervé Delingette, C.X., McVeigh, E.R., Ayache, N.: A computational framework for the statistical analysis of cardiac diffusion tensors: Application to a small database of canine hearts. IEEE Transactions in Medical Imaging 25(5), 612-625 (2006)

13. Powell, M.J.D.: Developments of newuoa for minimization without derivatives. Journal of Numerical Analysis, 1-16 (February 2008)

14. Reese, T.G., Weisskoff, R.M., Smith, R.N., Rosen, B.R., Dinsmore, R.E., Wedeen, V.J.: Imaging myocardial fiber architecture in vivo with magnetic resonance. Magnetic Resonance in Medicine 34, 786-791 (1995)

15. Rohmer, D., Gullberg, G.T.: A bloch-torrey equation for diffusion in a deforming media. Tech. rep., University of California (2006)

16. Sutton, M.G.S.J., Sharpe, N.: Left ventricular remodeling after myocardial infarction. Circulation: Cardiovascular Imaging 101, 2981-2988 (2000)

17. Tseng, W.Y.I., Wedeen, V.J., Reese, T.G., Smith, R.N., Halpern, E.F.: Diffusion tensor MRI of myocardial fibers and sheets: Correspondence with visible cut-face texture. Magnetic Resonance in Medicine 42, 17-31 (2003) 